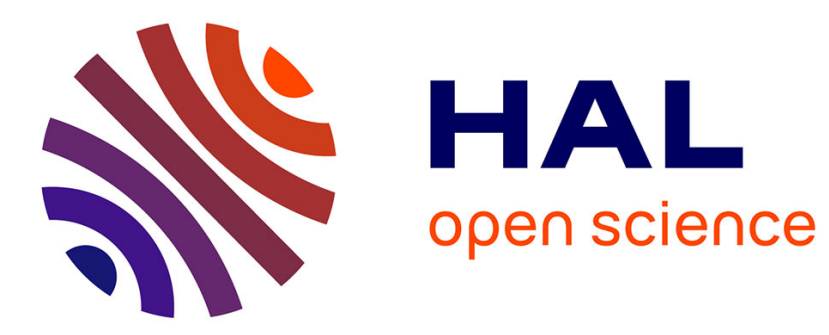

\title{
A flatness based speed control approach for merge behind operations
}

Thierry Miquel

\section{To cite this version:}

Thierry Miquel. A flatness based speed control approach for merge behind operations. AIAA GNC 2012, AIAA Guidance, Navigation and Control Conference, Aug 2012, Minneapolis, United States. pp xxxx, 10.2514/6.2012-4594. hal-00938804

\section{HAL Id: hal-00938804 \\ https://hal-enac.archives-ouvertes.fr/hal-00938804}

Submitted on 27 May 2014

HAL is a multi-disciplinary open access archive for the deposit and dissemination of scientific research documents, whether they are published or not. The documents may come from teaching and research institutions in France or abroad, or from public or private research centers.
L'archive ouverte pluridisciplinaire HAL, est destinée au dépôt et à la diffusion de documents scientifiques de niveau recherche, publiés ou non, émanant des établissements d'enseignement et de recherche français ou étrangers, des laboratoires publics ou privés. 


\title{
A Flatness Based Speed Control Approach For Merge Behind Operations
}

\author{
Thierry Miquel ${ }^{1}$ \\ Ecole Nationale de l'Aviation Civile, Toulouse, France
}

\begin{abstract}
Over the last few years, several concepts dealing the delegation to the flight crew of some tasks currently performed by the air traffic controllers have emerged. Among these new ideas, relative guidance has appeared to be capable to contribute to the enhancement of air traffic capacity though it raises difficult technical challenges. Indeed, this kind of maneuver appears difficult to perform manually, and may induce an excessive increase in flight crew workload, thus requiring new on-board automated functions. Some linear and nonlinear techniques have already been studied to design a feedback loop which performs automatically merging maneuvers and maintains station keeping behind a designated aircraft. The main contribution of this paper consists in the design of a feedback control loop using flatness control: this technique takes advantage of feedback linearization through a reference error between the ghost position of the leading aircraft and the current position of the trailing aircraft to provide a commanded speed to the autothrottle speed control system of the trailing aircraft. The robustness of the proposed design with respect to uncertainties in the dynamics of the leading aircraft and in the dynamics of the autothrottle speed control system of the trailing aircraft is achieved by the periodic update of the reference error. The reference error generation is detailed and simulation results are presented.
\end{abstract}

\section{Introduction}

$\mathrm{T}$ his paper addresses a specific aspect of Air Traffic Services (ATS), namely the achievement of an orderly and expeditious flow of air traffic. Terminal control area (TMA) air traffic controllers have to merge two or more streams into a single stream by means of radar vectoring and speed instructions. The task of establishing properly spaced landing sequences is quite demanding for air traffic controllers, especially under heavy traffic conditions. An example of typical flight path for arriving aircraft at an airport is depicted hereafter:

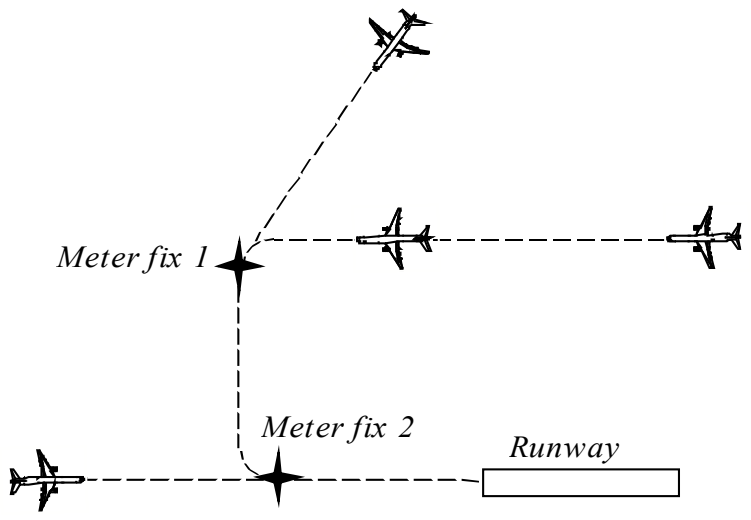

Fig. 1: Example of sequencing and merging operations for arriving aircraft at an airport

On the ground, an automation tool named Arrival Manager (AMAN) usually helps air traffic controllers to build a sequence of aircraft in order to safely and expeditiously land them ${ }^{[1]}$. Unfortunately, the airborne counterpart of the Arrival Manager, which could help the flight crew to merge its aircraft towards a meter fix according to a sequencing constraint, and ensure the station keeping after the meter fix, is not yet available. This yet to be designed

\footnotetext{
${ }^{1}$ Ecole Nationale de l'Aviation Civile, LARA Lab, thierry.miquel@enac.fr

Copyright $\odot 2011$ by ENAC. Published by the American Institute of Aeronautics and Astronautics, Inc., with permission.
} 
onboard function will ensure the merging and station keeping tasks behind a leading aircraft designated by the air traffic controller. This new function will use the information delivered by some surveillance and communication systems and should be included in future Flight Management System (FMS) capabilities. This will enable FMS to generate a trajectory with a prescribed delay relatively to another aircraft over a specified meter fixes.

Such a new capability onboard aircraft needs on-line information on the aircraft environment, and more specifically the knowledge of the leading aircraft position and velocity. In this respect, the Automatic Dependent Surveillance-Broadcast (ADS-B) is a potential key enabler to support these surveillance requirements ${ }^{[2]}$. Aircraft equipped with ADS-B capabilities broadcast their position, velocity and identification periodically (e.g. every second). Any neighboring aircraft capable of receiving those data will therefore be able to track the surrounding traffic.

Airborne Separation Assistance System (ASAS) uses ADS-B data to provide improved airborne surveillance in support of new operational procedures that increasingly delegate tasks from air traffic controllers to pilots ${ }^{[4]}$. The goal is to achieve a higher integration between the ground and the air. By definition proposed by ICAO ${ }^{[4]}$, ASAS is an airborne system that allows the flight crew to maintain separation between their aircraft and one or more other aircraft, and provides information concerning the surrounding traffic. ASAS on-board an aircraft interacts with the existing systems (particularly the FMS) or even with the installed equipment (particularly the displays); this may also require new equipments depending on the architecture. Not only the system, but also its utilization was defined by ICAO: an ASAS application is a set of operational procedures for air traffic controllers and flight crews, which makes use of the capabilities of airborne separation assistance systems to meet a clearly-defined operational objective.

In June 2001, the FAA/EUROCONTROL published a document ${ }^{[3]}$ within which 4 ASAS Application Categories were defined:

- Airborne Traffic Situational Awareness applications (ATSA)

- Airborne Spacing applications (ASPA)

- Airborne Separation applications (ASEP)

- Airborne Self-separation applications

Clearly, automatic merging and station keeping is an ASAS Airborne Spacing application (ASPA) for which controllers will issue new spacing instructions like Remain, Merge behind or Radar Vector Then Merge ${ }^{[3]}$. Flight crews will be asked through those new air traffic control instructions to establish and maintain a given time or distance relative to another aircraft and will use ASAS to achieve and maintain the required spacing. On the ground, controllers will monitor and provide separation: they will intervene if necessary to ensure that the actual spacing will not decrease below the separation standard; under that aspect, an ASPA instruction is similar to the instruction clearing an aircraft to fly at a specified flight level. This type of application is clearly in the scope of the European programme SESAR ${ }^{[5]}$ and the US programme NextGen. Those new technologies and procedures are part of a new paradigm that has the potential to completely transform the roles of air traffic controllers and pilots ${ }^{[3]}$.

In terms of operational benefits, this kind of application will relieve air traffic controllers of providing time consuming radar vectoring instructions to the trailing aircraft once the flight crew has accepted the relative guidance clearance. Thus, the expected benefit of such new capabilities is an increase of air traffic controller availability, which could result in increased air traffic efficiency and / or capacity. Enhancement airborne traffic situational awareness of the flight crew with associated safety benefits are also expected.

Preliminaries studies have mainly investigated the station keeping phase without taking into consideration the merging phase. This field is addressed for UAVs or military aircraft by means of linear and nonlinear techniques ${ }^{[6,7]}$. However, research for civil aircraft where safety and passenger comfort are crucial issues is still in its initial stage: in some early works, station keeping is either performed manually ${ }^{[8]}$ or by means of a proportional, integral and derivative (PID) control law ${ }^{[9,10]}$.

However, operations where the merging constraint is relaxed at the meter fix have to be tackled as part of the envisioned ASPA applications and may yield in more efficient operations than applying station keeping during the merging phase. The purpose of this paper is to investigate the design of a new autopilot mode dedicated to the achievement of a specified delay / distance between aircraft at a specified meter fix.

This paper considers a constant time delay as the separation objective at the meter fix; indeed it is more operational to express separation objective in terms of delay rather than distance in terminal maneuvering ${ }^{[11]}$. Eurocontrol Experimental Centre has investigated a specific method named Point Merge method for merging arrival flows in terminal area ${ }^{[14]}$. This method relies on the introduction of area navigation (RNAV, P-RNAV) and defines new route structures to revisit the merging of arrival flows. In the following, we consider that the merging and station keeping operations are achieved without change of the standard arrival routes. 
The paper is organized as follows: in the preliminaries, reference frame and dynamics of the error to be compensated are introduced. This leads to the very simple linear state space representation. The subsequent sections present the design of the flatness based speed control which sets the commanded speed applied to the autothrottle speed control system of the trailing aircraft. Illustrative examples are then presented in order to show the effectiveness of the proposed approach.

\section{Flatness Based Speed Control Design}

\section{A. Differentially flat system}

Flatness was originally introduced $1995^{[12]}$. Roughly speaking, a flat system is a square input/output system (i.e. a system having the same number of inputs and outputs) for which there exists an output vector such that all states and inputs can be expressed in terms of this output vector and its derivatives.

More precisely, a nonlinear system:

$$
\dot{x}=f(x, u)
$$

is differentially flat if one can find an output $z$ of the form:

$$
z=\zeta\left(x, u, \ldots, u^{(\rho)}\right)
$$

where $u^{(s)}$ denotes the s-th order derivative of $u$ with respect to time, and such that:

$$
\left\{\begin{array}{c}
x=\varphi_{0}\left(z, \ldots, z^{(s)}\right) \\
u=\varphi_{1}\left(z, \ldots, z^{(s)}, z^{(s+1)}\right)
\end{array}\right.
$$

Output $z$ is called flat output. In addition, system (1) is said Lie-Bäcklund equivalent to the following system (called trivial system), where vector $v$ is the new input, also called pseudo control:

$$
v=z^{(s+1)}
$$

Imposing a given arbitrary trajectory to $z$ yields a trajectory for all the system variables $x$ and $u$, without integrating any differential equation. Remark that the time derivatives involved in the above formulas do not imply to take derivatives of noisy signals since it involves precomputed open-loop time functions.

Fig. 2 shows the principle of a feedback loop for flatness based control: $\Sigma$ denotes the system to be controlled and described by the dynamics $\dot{x}=f(x, u), \Sigma^{-1}$ its inverse from which the open loop control $u_{o l c}=\varphi_{1}\left(z_{r}, \ldots, z_{r}^{(s)}, z_{r}^{(s+1)}\right)$ is computed, and $K$ a feedback gain which enables the control $u$ to retrieve from errors between the measured state vector $x$ and the reference trajectory $z_{r}$.

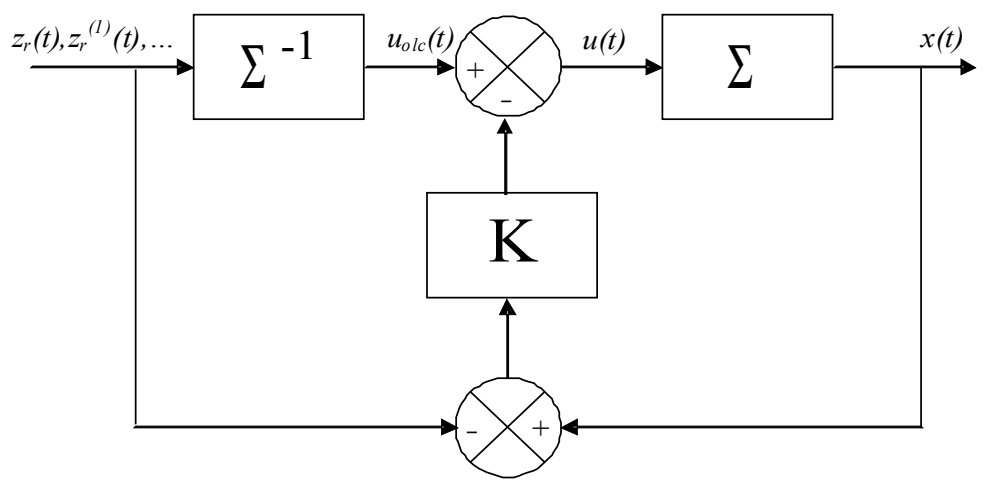

Fig. 2: Feedback loop for flatness based control

\section{B. Reference frame and error dynamics}

The reference frame is affixed to the trailing aircraft. From Fig. 3, the following notations are adopted:

- Subscript $L$ stands for ghost position of the leading aircraft, that is the position of the leading aircraft delayed by the required delay which should be achieved at the meter fix $P$. This delay is set be the air traffic controller;

- $\quad$ Subscript stands $F$ for the current position of trailing aircraft (follower);

- $d_{L}$ stands for the distance between the ghost position of the leading aircraft and the meter fix $P$, whereas $d_{F}$ stands for the current distance between the trailing aircraft and the meter fix. 


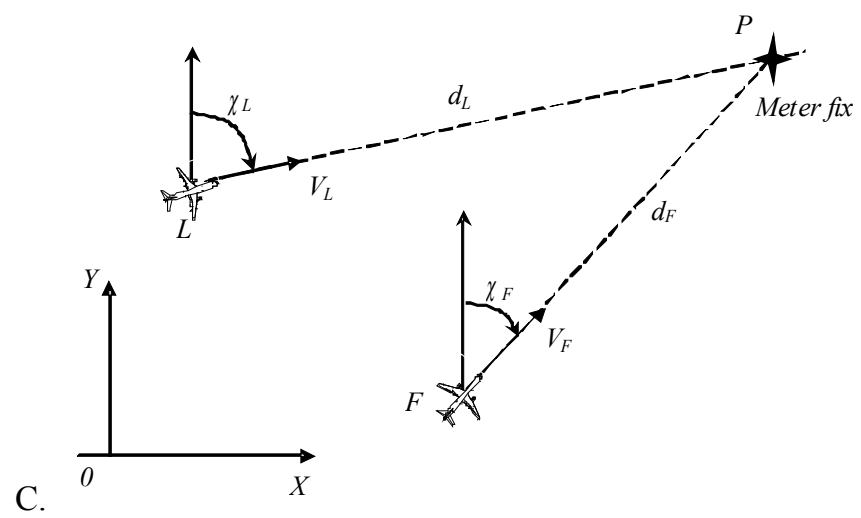

Fig. 3: Reference frame and notations

The dynamics of the error $e$ between the ghost position of the leading aircraft and the current position of the trailing aircraft is simply given by:

$$
\dot{e}=V_{L}-V_{F}
$$

In the following, the difference $V_{L}-V_{F}$ will be used as a pseudo control, denoted $u$, to control the dynamics of the error $e$ :

$$
u=V_{L}-V_{F} \Rightarrow \dot{e}=u
$$

The linear model which represents the dynamics of the error $e$ between the ghost position of the leading aircraft and the current position of the trailing is then very simple: it is basically an integrator.

\section{Feedback control using flat outputs}

It is clear that the error $e$ between the ghost position of the leading aircraft and the current position of the trailing aircraft is a flat output of system (6).

It is assumed in the following that an autopilot function dealing with airspeed control is available on-board the trailing aircraft. The purpose of the relative guidance control system is then to set the commanded airspeed such that the trailing aircraft reaches the meter fix at the same time than the ghost position of the leading aircraft; then station keeping has to be maintained behind the leading aircraft.

To achieve global stability, a Lyapunov based design is used: assume that there exists a scalar function $L$ of the state $e$, with continuous first order derivatives such that ${ }^{[13]}$ :

- $\quad L(e)$ is positive definite

- $L(e) \rightarrow \infty$ as $\|e\| \rightarrow \infty$

- $\quad \dot{L}(e)$ is negative definite

The following quadratic candidate Lyapunov function will be used, where $e_{r}$ stands for the reference error between the ghost position of the leading aircraft and the current position of the trailing aircraft:

$$
L(e)=\frac{1}{2}\left(e-e_{r}\right)^{2}
$$

The pseudo control $u$ has to be designed such that the reference error $e_{r}$ is tracked by system (6). Taking time derivative and using (6) yields:

$$
\dot{L}(e)=\left(e-e_{r}\right)\left(\dot{e}-\dot{e}_{r}\right)=\left(e-e_{r}\right)\left(u-\dot{e}_{r}\right)
$$

$\dot{L}(e) \quad$ will be negative definite by choosing the pseudo control $u$ as follows, where $k_{p}$ is a gain to be set by the designer:

$$
u=\dot{e_{r}}-k_{p}\left(e-e_{r}\right) \Rightarrow \dot{L}(e)=-k_{p}\left(e-e_{r}\right)^{2}
$$

The commanded airspeed applied to the autopilot function dealing with airspeed control is then given by:

$$
u \stackrel{\text { def }}{=} V_{L}-V_{F} \Rightarrow V_{F}=V_{L}-u=V_{L}-\dot{e}_{r}+k_{p}\left(e-e_{r}\right)
$$

The error $e(t)$ between the positions of ghost aircraft and the trailing aircraft results from the integration of (5) 
but can be directly obtained without any integration thanks to ADS-B data.

When compared with Fig. 2, the term $V_{L}-\dot{e}_{r}$ represents the open loop control $u_{\text {olc }}(t)$, whereas $k_{p}$ is the feedback gain $K$ which enables the control to retrieve from errors between the measured error $e$ and the reference error $e_{r}$.

The problem of the relative guidance is then viewed as a problem where explicit trajectory generation of reference $e_{r}$ is required. Since the behavior of flat systems is determined by their flat outputs, the use of flatness to design relative guidance controller is quite natural. Indeed, trajectory in the flat output space can be planned and then mapped to appropriate inputs.

\section{E. Reference error computation}

As far as the flight plan of the leading aircraft is not known by the trailing aircraft, the duration $T$ needed by the ghost position of the leading aircraft to reach the meter fix is simply estimated by the ratio between distance $d_{L}$ to the meter fix and the airspeed $V_{L}$ of the leading aircraft ghost position:

$$
T \approx \frac{d_{L}(0)}{V_{L}}
$$

Referring to Fig. 3, and assuming a constant airspeed $V_{L}$ for the leading aircraft, the reference error $e_{r}(t)$ between the positions of ghost aircraft and the trailing aircraft is chosen as follows, where $l(t)$ denotes the reference distance of the trailing aircraft to the meter fix:

$$
\left\{\begin{array} { c } 
{ e _ { r } ( t ) \stackrel { \text { def } } { = } d _ { F } ( 0 ) - l ( t ) - ( d _ { L } ( 0 ) - V _ { L } t ) } \\
{ \dot { e } _ { r } \stackrel { \text { def } } { = } V _ { L } - V _ { r } }
\end{array} \text { where } \left\{\begin{array}{c}
l(0) \stackrel{\text { def }}{=} 0 \\
i(t) \stackrel{\text { def }}{=} V_{r}(t) \\
e(0)=d_{F}(0)-d_{L}(0)
\end{array}\right.\right.
$$

Then, the control law (10) reduces to the following expression, where $d_{F}(t)$ denotes the actual distance between the trailing aircraft and the merging point and $V_{F}$ the commanded airspeed applied to the autothrottle speed control system of the trailing aircraft:

$$
\left\{\begin{array}{c}
e(t) \stackrel{\text { def }}{=} d_{F}(t)-\left(d_{L}(0)-V_{L} t\right) \\
e_{r}(t) \stackrel{\text { def }}{=} d_{F}(0)-l(t)-\left(d_{L}(0)-V_{L} t\right) \Rightarrow V_{F}=V_{L}-\dot{e_{r}}+k_{p}\left(e-e_{r}\right)=V_{r}+k_{p}\left(d_{F}(t)-d_{F}(0)+l(t)\right) \\
\dot{e_{r}} \stackrel{\stackrel{\text { def }}{=} V_{L}-V_{r}}{ }
\end{array}\right.
$$

As far as it is desired that trailing aircraft arrives at the meter fix at the same time than the ghost position of the leading aircraft, and with the same speed, the reference error $e_{r}$ between the ghost position and the trailing aircraft position has to be computed such that the following constraints are satisfied:

$$
\left\{\begin{array}{c}
e_{r}(T)=0 \\
\dot{e}_{r}(T)=V_{L}-V_{r}(T) \stackrel{\text { def }}{=} 0
\end{array}\right.
$$

In the following, we will replace time $t$ by the dimensionless parameter $\tau$ which is defined by the ratio between actual time $t$ and the estimated duration $T$ of the merging maneuver (see (11)):

$$
0 \leq \tau \stackrel{\text { def }}{=} \frac{t}{T} \leq 1
$$

Two options to set the reference length $l(\tau)$ of the trailing aircraft to the meter fix are presented hereafter.

- The first option only consider the constraints presented in (14):

$$
l(\tau)=T\left(a_{0} \tau+\frac{a_{2}}{\sqrt{b}}(\operatorname{atan}(\sqrt{b}(\tau-1))+\operatorname{atan}(\sqrt{b}))\right)
$$

Where parameters $a_{0}, a_{2}$ and $b$ are free parameters. Time derivation of the preceding equation leads to the expression of the trailing aircraft's reference airspeed:

$$
V_{r}(\tau)=a_{0}+\frac{a_{2}}{b(\tau-1)^{2}+1}
$$

In order to satisfy the constraints presented in (14), parameters $a_{0}, a_{2}$ and $b$ shall satisfy the following relationships: 


$$
\left\{\begin{array}{c}
e_{r}(1) \stackrel{\text { def }}{=} 0 \Leftrightarrow l(1)=d_{F}(0) \Leftrightarrow a_{0}+\frac{\operatorname{atan}(\sqrt{b})}{\sqrt{b}} a_{2}=\frac{d_{F}(0)}{T} \\
\dot{e}_{r}(1) \stackrel{\text { def }}{=} V_{L}-V_{r}(1)=0 \Leftrightarrow a_{0}+a_{2}=V_{L}
\end{array}\right.
$$

Assuming that parameter $b$ is already set, parameters $a_{0}$ and $a_{2}$ can easily be computed from the preceding equations.

- The second option consider the constraints presented in (14) and also sets the initial value of the reference speed at the current speed of the trailing aircraft. We consider the following expression for reference length $l(\tau)$, where parameters $a_{0}, a_{1}, a_{2}$ and $b$ are free parameters:

$$
l(\tau)=T\left(a_{0} \tau+\frac{a_{1}}{\sqrt{b}} \operatorname{atan}(\sqrt{b} \tau)+\frac{a_{2}}{\sqrt{b}}(\operatorname{atan}(\sqrt{b}(\tau-1))+\operatorname{atan}(\sqrt{b}))\right)
$$

Time derivation of the preceding equation leads to the expression of the trailing aircraft's reference airspeed:

$$
V_{r}(\tau)=a_{0}+\frac{a_{1}}{b \tau^{2}+1}+\frac{a_{2}}{b(\tau-1)^{2}+1}
$$

In order to set the initial value of the reference speed at the current speed of the trailing aircraft and to satisfy the constraints presented in (14), parameters $a_{0}, a_{1}, a_{2}$ and $b$ shall satisfy the following relationships:

$$
\left\{\begin{array}{c}
V_{r}(0) \stackrel{\text { def }}{=} V_{0} \Leftrightarrow a_{0}+a_{1}+\frac{a_{2}}{b+1}=V_{0} \\
e_{r}(1) \stackrel{\text { def }}{=} 0 \Leftrightarrow l(1)=d_{F}(0) \Leftrightarrow a_{0}+\frac{\operatorname{atan}(\sqrt{b})}{\sqrt{b}}\left(a_{1}+a_{2}\right)=\frac{d_{F}(0)}{T} \\
\dot{e}_{r}(1) \stackrel{\text { def }}{=} V_{L}-V_{r}(1)=0 \Leftrightarrow a_{0}+\frac{a_{1}}{b+1}+a_{2}=V_{L}
\end{array}\right.
$$

Assuming that parameter $b$ is already set, parameters $a_{0}, a_{1}$ and $a_{2}$ can easily be computed from the preceding equations.

\section{Illustrative examples}

\section{A. Scenarios}

In this section, two scenarios are designed in order to illustrate the properties of the control laws previously designed. The meter fix point $P$ is situated at $O N M$.

For both scenarios, the ghost position of the leading aircraft starts at $d_{L}(0)=-25 N M$ with initial airspeed $V_{L}(0)=220 \mathrm{kts}$. It is supposed to broadcast its data every second ( $A D S-B$ basic assumption). The initial position of the trailing aircraft is $d_{F}(0)=-30 \mathrm{NM}$ with an initial airspeed $V_{F}(0)=210 \mathrm{kts}$.

In the first scenario, the ghost airspeed of the leading aircraft remains constant, whereas in the second scenario the ghost airspeed of the leading aircraft decreases towards $120 \mathrm{kts}$ with a deceleration of $0.01 \mathrm{~g}$.

While the ghost position of the leading aircraft has not reached the meter fix point $P$, the speed control feedback loop of the trailing aircraft is in the merge behind mode: the reference errors $e_{r}(t)$ and $\dot{e}_{r}(t)$ are computed according to the two options previously presented, and the commanded airspeed $V_{F}$ which feeds the autopilot is given by (10):

$$
V_{F}=V_{L}-u=V_{L}-\dot{e}_{r}+k_{p}\left(e-e_{r}\right)
$$

Once the ghost position of the leading aircraft has passed the meter fix point $P$, the speed control feedback loop switches to the remain behind mode where the reference errors $e_{r}(t)$ and $\dot{e}_{r}(t)$ are set to zero. In such mode, the commanded airspeed $V_{F}$ is simply:

$$
V_{F}=V_{L}+k_{p} e
$$

In both modes the gain $k_{p}$ has been set to 50 . In addition the robustness of the proposed design with respect to uncertainties in the dynamics of the leading aircraft and the dynamics of the speed control autopilot is achieved by the periodic update of the reference error: every $30 \mathrm{sec}$ for both options.

The dynamics of the speed control autopilot of the trailing aircraft is modeled by a second order linear system with a damping ratio $m$ of 0.7 and a natural frequency $w_{0}$ of $0.5 \mathrm{rad} / \mathrm{sec}$. Thus the relationship between then actual speed $V$ of the trailing aircraft and the commanded airspeed $V_{F}$ is given by: 


$$
\ddot{V}=-2 \mathrm{mw}_{0} \dot{V}-w_{0}^{2}\left(V-V_{F}\right)
$$

It is worth noticing that the acceleration of the trailing aircraft has been limited to $0.05 \mathrm{~g}$.

Positions $x_{L}$ of the ghost and $x_{F}$ of the trailing aircraft are linked to speed $V_{L}$ and $V$ through an integration:

$$
\left\{\begin{array}{c}
\dot{x}_{L}=V \\
\dot{x_{F}}=V
\end{array}\right.
$$

The two flatness based speed control design to compute the commanded speed $V_{F}$ are compared against a design where the commanded speed $V_{F}$ is simply computed as proportional to the actual error between the ghost position of the leading aircraft and the current position of the trailing aircraft, that is as in (23) even during the merge behind phase.

\section{B. Simulation results}

All the scenarios are summarized and labeled in the following table:

\begin{tabular}{|c|c|c|c|}
\hline & $\begin{array}{c}\text { Proportional based speed } \\
\text { control - no reference } \\
\text { error }\end{array}$ & $\begin{array}{c}\text { Flatness based speed } \\
\text { control: reference error as } \\
\text { defined by (17) }\end{array}$ & $\begin{array}{c}\text { Flatness based speed } \\
\text { control: reference error as } \\
\text { defined by (13) }\end{array}$ \\
\hline Constant velocity of ghost & Scenario 01 & Scenario 02 & Scenario 03 \\
\hline Ghost deceleration to $120 \mathrm{kts}$ & Scenario 11 & Scenario 12 & Scenario 13 \\
\hline
\end{tabular}

Computations have been achieved thanks to Scilab, the open source platform for numerical computation ${ }^{[15]}$.

Fig. 4 presents commanded true airspeed and actual airspeed of the trailing aircraft as well as the airspeed of the ghost for all scenarios whereas Fig. 5 presents the positions of ghost and trailing aircraft for all scenarios. Speeds are expressed in kts, positions in $N M$ and time in seconds.

As expected, the scenarios involving a flatness based speed control enable the trailing aircraft to reach the meter fix at the same time than the ghost position of the leading aircraft, whereas the scenario involving a proportional based speed control leads the trailing aircraft to catch the ghost position before the meter fix. In terms of airspeed, the scenarios involving a flatness based speed control leads to lower speed deviation and speed command when compared to the scenario involving a proportional based speed control. Thus the greater complexity of the flatness based speed control due the fact that time explicitly appears in the control law and to the need of periodically update the computation of the reference error are compensated by higher performances for merge achievement. 


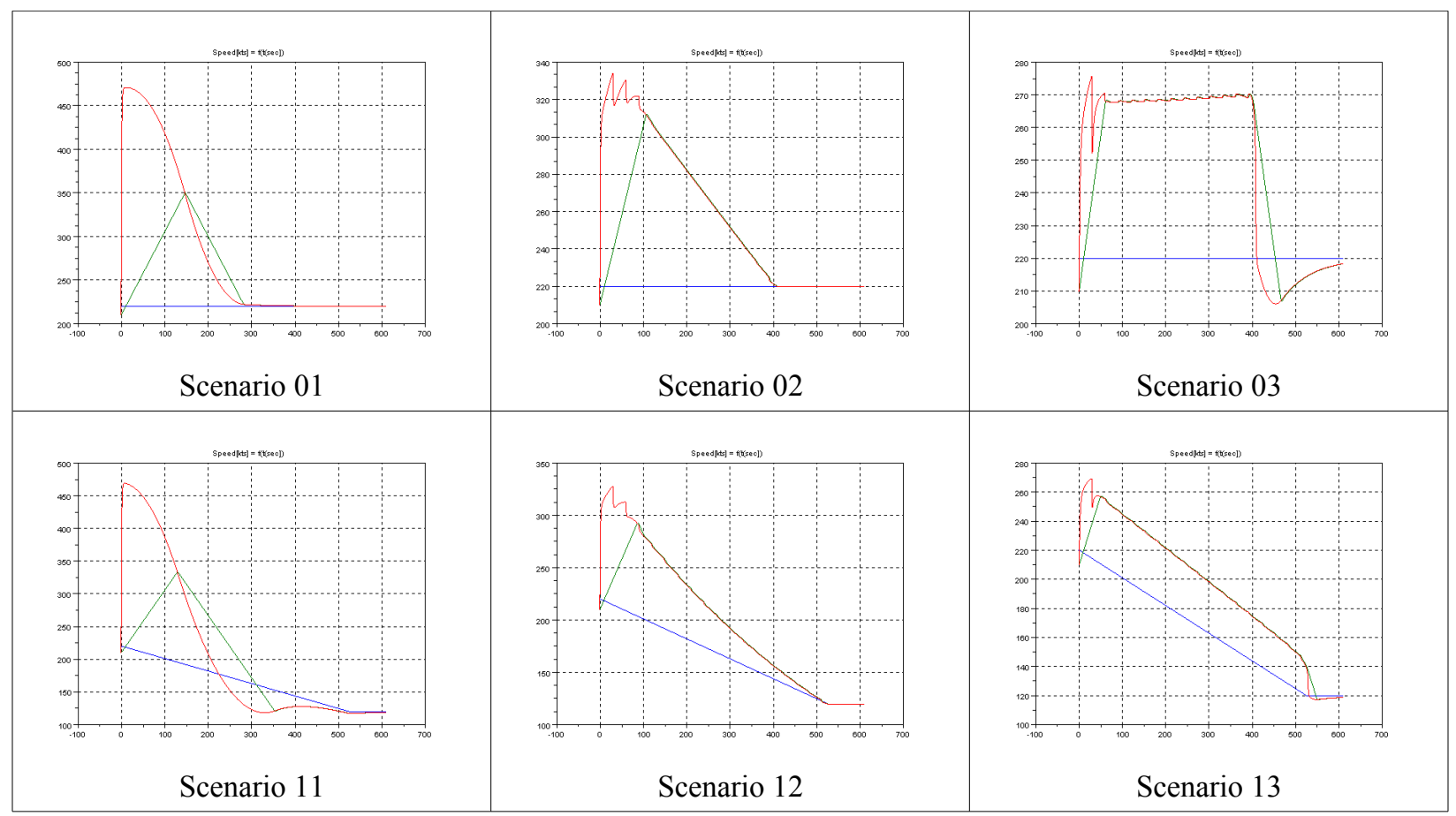

Fig. 4: Speeds of trailing aircraft (commanded in red and actual in green) and ghost (blue)

Fig. 5 presents the positions of ghost and trailing aircraft for all scenarios:

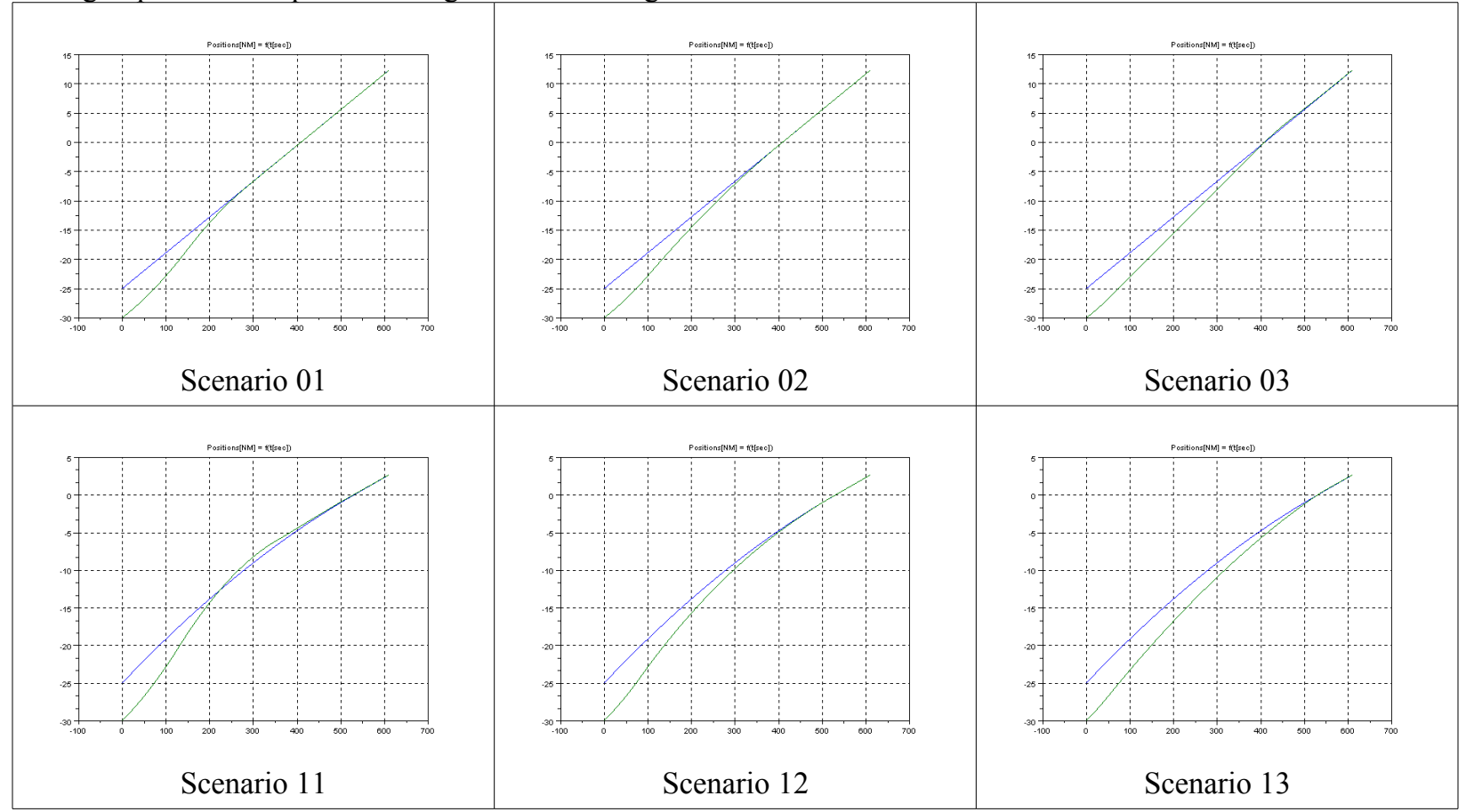

Fig. 5: Positions of trailing aircraft (green) and ghost (blue) for all scenarios 


\section{Conclusion}

In this paper, the design of a new autopilot mode dedicated to the achievement of a specified delay between commercial aircraft at a specified meter fix has been considered.

This envisioned new capability on-board commercial aircraft takes advantage of the Automatic Dependent Surveillance-Broadcast $(A D S-B)$ and provides new perspectives to potentially increase air traffic control efficiency. It could be the airborne counterpart of the ground based arrival manager.

The proposed approach is based on flatness control: this technique takes advantage of feedback linearization through a reference error between the ghost position of the leading aircraft and the current position of the trailing aircraft. It provides a commanded speed to the speed control autopilot of the trailing aircraft. The robustness of the proposed design with respect to uncertainties in the dynamics of the leading aircraft and in the dynamics of the speed control autopilot of the trailing aircraft is achieved by the periodic update of the reference error. When compared to a proportional based control, the greater complexity of the flatness based control due the fact that time explicitly appears in the control law and to the need of periodically update the computation of the reference error are compensated by higher performances for merge operations.

This paper has considered a 1 dimensional movement. The extension to the 3 dimensional space can easily be done by considering the curvilinear abscissa flown by aircraft.

Simulation results illustrates the efficiency of the proposed design when compared to a proportional based speed control depending only of the actual error. Nevertheless, the way to accurately get the time at which the meter fix is reach by the leading aircraft should be tackled. Furthermore, additional studies in terms of operational scenarios and taking into account the vertical motion of the aircraft as well as the effect of wind are needed in order to refine and validate the proposed design.

\section{References}

1 Kayton M., Fried W. R., Avionics navigation systems, second edition, John Wiley \& Sons, New York, 1997

2 Hoffman E., Ivanescu D., Shaw C., Zeghal K., Effect of Automatic Dependent Surveillance Broadcast (ADS-B) transmission quality on the ability of aircraft to maintain spacing in sequence, Air Traffic Control Quaterly, Special Issue: Aircraft Surveillance Applications of ADS-B, 2003

3 PO-ASAS, Principles of Operation for the Use of Airborne Separation Assurance Systems Version 7.1, FAA/Eurocontrol Co-operative R\&D, Eurocontrol, Brussels, 2001

4 ICAO, Report Of Committee A to the Conference On Agenda Item 1, AN-Conf/11-WP/190, The Eleventh ICAO Air Navigation Conference, Montreal, Canada, 2003

5 SESAR Consortium, The ATM Target Concept - D3, DLM-0612-001-02-00a, 2007, (http://www.sesarconsortium.aero/deliv3.php)

6 Pachter M., D'Azzo JJ., Proud AW., Tight formation flight control, Journal of Guidance, Control, and Dynamics, Vol. 24, pp246-254, 2001

7 Singh SN., Zhang R., Chandler P., Banda S., Decentralized nonlinear robust control of UAVs in close formation, International Journal of Robust and Nonlinear Control, Vol. 13 pp1057-1078, 2003

8 Agelii M., Olausson C., Flight deck simulations of station keeping, ATM R\&D seminar, Santa Fe, paper no. 17,2001

9 Vinken P., Hoffman E., Zeghal K., Influence of speed and altitude profile on the dynamics of in-trail following aircraft, Proceeding of AIAA Guidance Navigation and Control Conference. Denver, USA, Paper No. 20004362,2000

10 Hoffman E., Ivanescu D., Shaw C., Zeghal K., Analysis Of Spacing Guidance For Sequencing Aircraft On merging Trajectories, 21st Digital Avionics Systems Conference, Irvine, California, October 2002

11 Agelii M., Olausson C., Flight deck simulations of station keeping, ATM R\&D seminar, Santa Fe, paper no. 17,2001

12 Fliess M., Lévine J., Martin P., Rouchon P., Flatness and Defect of Non-linear Systems: Introductory Theory And Examples, International Journal of Control, 61:1327-1361, 1995

13 Khalil H.K., Nonlinear Systems (3rd Edition), Prentice Hall; 3 edition, 2001

14 Boursier L., Favennec B., Hoffman E., Trzmiel A., Vergne F., Zeghal K., Merging Arrival Flows Without Heading Instructions, 7th USA/Europe Air Traffic Management R\&D Seminar, Spain, July 2007

15 Scilab, the open source platform for numerical computation (http://www.scilab.org/) 